\title{
Phenotyping Sunflower Genetic Resources for Sclerotinia Head Rot Response: Assessing Variability for Disease Resistance Breeding
}

C. V. Filippi and J. E. Zubrzycki, Instituto de Biotecnología, Centro de Investigaciones en Ciencias Veterinarias y Agronómicas (CICVyA), Instituto Nacional de Tecnología Agropecuaria (INTA), Nicolas Repetto y Los Reseros s/n (1686), Hurlingham, Buenos Aires, Argentina; J. A. Di Rienzo, Facultad de Ciencias Agropecuarias, Universidad Nacional de Córdoba, Ing Agr. Felix Aldo Marrone 746 (5000), Córdoba, Argentina; F. Quiroz, Estación Experimental Agropecuaria INTA Balcarce, Ruta 226 Km 73.5 (7620), Balcarce. Buenos Aires, Argentina; C. M. Fusari, Instituto de Biotecnología, Centro de Investigaciones en Ciencias Veterinarias y Agronómicas (CICVyA), Instituto Nacional de Tecnología Agropecuaria (INTA), Nicolas Repetto y Los Reseros s/n (1686), Hurlingham, Buenos Aires, Argentina; and Max Planck Institute of Molecular Plant Physiology, Am Mühlenberg 1 (14476), Potsdam, Germany; D. Alvarez, Estación Experimental Agropecuaria INTA Manfredi, Ruta 9 Km 636 (5988), Manfredi, Córdoba, Argentina; C. A. Maringolo, Estación Experimental Agropecuaria INTA Balcarce, Ruta 226 Km 73.5 (7620), Balcarce. Buenos Aires, Argentina; D. Cordes, Estación Experimental Agropecuaria INTA Manfredi, Ruta 9 Km 636 (5988), Manfredi, Córdoba, Argentina; A. Escande, Estación Experimental Agropecuaria INTA Balcarce, Ruta 226 Km 73.5 (7620), Balcarce. Buenos Aires, Argentina; H. E. Hopp, Instituto de Biotecnología, Centro de Investigaciones en Ciencias Veterinarias y Agronómicas (CICVyA), Instituto Nacional de Tecnología Agropecuaria (INTA), Nicolas Repetto y Los Reseros s/n (1686), Hurlingham, Buenos Aires, Argentina; and Facultad de Ciencias Exactas y Naturales Universidad de Buenos Aires, Intendente Güiraldes 2160, (1428), Ciudad Autónoma de Buenos Aires, Argentina; R. A. Heinz and V. V. Lia, ${ }^{\dagger}$ Instituto de Biotecnología, Centro de Investigaciones en Ciencias Veterinarias y Agronómicas (CICVyA), Instituto Nacional de Tecnología Agropecuaria (INTA), Nicolas Repetto y Los Reseros s/n (1686), Hurlingham, Buenos Aires, Argentina; Facultad de Ciencias Exactas y Naturales Universidad de Buenos Aires, Intendente Güiraldes 2160, (1428), Ciudad Autónoma de Buenos Aires, Argentina; and Consejo Nacional de Investigaciones Científicas y Técnicas-CONICET; and N. B. Paniego, Instituto de Biotecnología, Centro de Investigaciones en Ciencias Veterinarias y Agronómicas (CICVyA), Instituto Nacional de Tecnología Agropecuaria (INTA), Nicolas Repetto y Los Reseros s/n (1686), Hurlingham, Buenos Aires, Argentina; and Consejo Nacional de Investigaciones Científicas y Técnicas-CONICET

\begin{abstract}
Sclerotinia head rot (SHR) is one of the most serious constraints to sunflower (Helianthus annuus L. var. macrocarpus) production worldwide. Here, we evaluated the response to SHR in a sunflower inbred panel from a large INTA germplasm collection, consisting of 137 inbred lines (ILs). Field trials were performed over five consecutive seasons using a twicereplicated randomized complete-block design. Disease incidence, disease severity, incubation period, and area under disease progress curve for disease incidence and severity were determined after controlled inoculation with the pathogen. Statistical analysis using mixed-effect models detected significant differences among ILs for all variables $(P<0.001)$. In addition,

principal component analysis (PCA) and distance-based methods were used to classify the ILs according to their response to SHR, with ILs ALB2/5261 and 5383 emerging as the most resistant. Broad-sense heritability estimates ranged from $20.64 \%$ for disease severity to $10.58 \%$ for incubation period. The ample phenotypic variability of our collection, along with the moderate heritability estimates, highlight the importance of molecular breeding approaches to gain new insights into the genetic basis of sunflower resistance to SHR. The exhaustive phenotypic characterization presented here provides a reliable set of variables to comprehensively evaluate the disease and identifies two new sources of resistance to SHR.
\end{abstract}

Cultivated sunflower (Helianthus annuus L. var. macrocarpus) is one of the most important oilseed crops, covering 25 million ha worldwide. The total annual production in the world is about 36 million metric tons, mainly concentrated in the Russian Federation, Ukraine, the European Union, and Argentina, which is the fourth largest producer and the third oil exporter (www.sunflowernsa. com). However, there is a large gap between the potential and actual yields, mainly due to biotic and abiotic constraints.

Sclerotinia head rot (SHR) is a major disease of sunflower, causing serious damage to production in almost all the sunflower-growing areas of the world (Boland and Hall 1994). Achieving control of this disease is challenging for three main reasons: Sclerotinia sclerotiorum (Lib.) de Bary, its etiological agent, is a necrotrophic fungus able to survive for many years in the soil (Clarkson et al. 2013); it can

${ }^{\dagger}$ Corresponding author. E-mail: lia.veronica@inta.gob.ar

V. V. Lia and N. B. Paniego are equal contributors.

*The $\boldsymbol{e}$-Xtra logo stands for "electronic extra" and indicates that six supplementary tables and three supplementary figures are published online.

Accepted for publication 18 June 2017.

@ 2017 The American Phytopathological Society infect a wide host range, from annual plants to woody crops (Boland and Hall 1994); and effective therapeutic treatments are not yet available (Van and Miller 2004). In this scenario, the development of resistant lines by pyramiding QTL and candidate genes, using both classical and molecular breeding tools, appears to be one of the most promising strategies for crop protection. Until recently, biparental mapping was the method of choice to establish a link between phenotype and genotype and to identify the genomic regions underlying quantitative variation of complex traits. Nowadays, interest is shifting to the use and mining of germplasm collections, through association mapping approaches (Zhu and Salmeron 2007). Regardless of the breeding approach, phenotyping of traits related to SHR resistance necessarily involves multienvironmental trials with a large number of individuals.

A key aspect of screening for plant disease resistance is determining which are the most suitable variables and methods for scoring the disease. In sunflower, SHR response has usually been evaluated using variables such as disease severity (proportion of diseased host tissue), disease incidence (number or proportion of diseased plants), and incubation period (number of days until onset of symptoms) (e.g., Bert et al. 2002, 2004; Castaño and Giussani 2009; Vear 2004; Yue et al. 2008). Previous studies on SHR have reported different resistance QTL depending on whether disease severity, disease incidence or incubation period were being considered, suggesting that each of these variables capture different aspects of resistance (Yue et al. 2008). 
From a methodological standpoint, a variety of methods have been employed for screening sunflower lines and hybrids (Baldini et al. 2002; Castaño et al. 1993; Van and Miller 2004; Vear and Tourvieille de Labrouhe 1984, 1988). Among them, the application of ascospore suspensions on the floral surface is one of the most commonly used testing procedures as it mimics the natural epidemiological cycle quite closely and allows measurement of resistance throughout the whole pathogen's development on the host (Vear and Tourvieille de Labrouhe 1988).

As with the above mentioned experimental procedures, the development of adequate statistical models capable of providing accurate adjusted phenotypic means is also critical. Until now, the statistical analysis of phenotypic data of sunflower resistance to SHR has mainly relied on analysis of variance (ANOVA) (Baldini et al. 2002; Castaño et al. 1993; Mestries et al. 1998; Rönicke et al. 2005; Yue et al. 2008). However, the classical ANOVA models, based on fixed effects and restricted to the assumptions of independence and homoscedasticity of the error terms, cannot cope with either incomplete data sets or more complex scenarios (e.g., incomplete blocks, heteroscedastic and/or correlated errors). Moreover, the classical models are also inappropriate when working with non-normally distributed variables. In this context, estimation and hypothesis testing based on extended and generalized linear mixed models emerge as the most suitable choice for the analysis of SHR resistance data.

The Active Germplasm Bank of the Manfredi Experimental Station (AGB-IM)-Instituto Nacional de Tecnología Agropecuaria (INTA) holds ca. 1,200 accessions of cultivated sunflower, with representatives from a broad range of geographic origins, and a large proportion of locally developed cultivars. This "Argentinean germplasm" has a distinctive genetic constitution and is well adapted to the highly variable environmental conditions of the sunflower cultivation areas of Argentina, i.e., from very hot in the semiarid region of the Chaco Province to rather cold and humid in the southern Pampas (Filippi et al. 2015; Moreno et al. 2013).

In seeking to achieve a balance between genetic diversity and local adaptation, 137 inbred lines (ILs) from the AGB-IM were selected to develop an association mapping population. This inbred line panel (ILP) is currently used by the sunflower breeding program of INTA to detect useful genetic variation for a number of agronomically important traits, including resistance to SHR (Fusari et al. 2012). Molecular diversity assessment of this collection revealed the existence of three different genetic groups, with the maintainer/restorer status being the most prevalent characteristic associated with group delimitation (Filippi et al. 2015).

In this study, we used controlled inoculation and replicated field trials over five years to evaluate resistance to SHR in the 137-line ILP. The main goals of this work were to evaluate disease response using a comprehensive set of variables and to identify new genetic sources of SHR resistance for sunflower breeding.

\section{Materials and Methods}

Plant material and experimental field plot design. The sunflower ILP used for this study was composed of 137 ILs: 66 ILs from the sunflower breeding program of INTA for disease resistance, 54 ILs from the sunflower breeding program of INTA for abiotic stress tolerance, and 17 public ILs of diverse genetic background. The complete list of ILs and their history in sunflower breeding programs is presented in Supplementary Table S1. For more information about pedigree, country of origin, and other agronomical characteristics, see Filippi et al. (2015). All the inbred lines included in this study are available upon request from the AGB-IM.

Field trials (FTs) were conducted at agricultural experimental station (AES) INTA Balcarce $\left(37^{\circ} 50^{\prime} 0^{\prime \prime}\right.$ S, 58 $15^{\prime} 33^{\prime \prime} \mathrm{W}$, Province of Buenos Aires, Argentina) during growing seasons 2009-10 (sowing date 11 December 2009), 2010-11 (sowing date 6 December 2010), 2011-12 (sowing date 5 December 2011), 2012-13 (sowing date 6 December 2012), and 2013-14 (sowing date 9 December 2013). The first three and the last two FTs were performed under nonirrigation and irrigation regimes, respectively, with plants being sprayirrigated daily, at noon, for $20 \mathrm{~min}$.
To break seed dormancy, seeds were incubated with a solution of gibberellic acid $\left(\mathrm{GA}^{3}\right)(100 \mathrm{ppm})$ for $60 \mathrm{~min}$, followed by incubation at $10^{\circ} \mathrm{C}$ until sowing. After treatment, seeds were planted by hand in Typic Argiudoll soil containing 5\% organic matter at pH 6.2.

The FTs were conducted in a randomized complete block design with two blocks. Each experimental unit was one row $9.0 \mathrm{~m}$ long by $0.7 \mathrm{~m}$ wide, with a planting distance of $0.25 \mathrm{~m}$, resulting in 36 plants per row.

Fungal isolates. $S$. sclerotiorum sclerotia derived from naturally and experimentally infected plants were collected at AES INTA Balcarce every year and used for ascospore production according to Escande et al. (2002). Briefly, sclerotia were exposed to $-18 \pm 2{ }^{\circ} \mathrm{C}$ for 7 days and then cultivated in humid pasteurized Typic Argiudoll soil in darkness until germination, followed by incubation at $16^{\circ} \mathrm{C}$ under continuous illumination of 2,500 lux. Mature apothecia were collected in Petri dishes and incubated for $4 \mathrm{~h}$ to favor ascospore release. Ascospores were stored in plastic plates at $-18^{\circ} \mathrm{C}$ until inoculation. To produce inoculum, the spores were washed from the plates with $10 \mathrm{ml}$ sterile water, and adjusted to a concentration of 2,500 ascospores $/ \mathrm{ml}$ with a Neubauer hemocytometer. Fresh inoculum was prepared at each inoculation date immediately before use.

Inoculation of inbred lines. All plants were inoculated with the pathogen at the R5.2 flowering stage of the scale of Schneiter and Miller (1981). Capitula were inoculated using a portable hand sprayer with $1 \mathrm{ml}$ of inoculum $(2,500$ ascospores $/ \mathrm{ml})$ following the method of Tourvieille de Labrouhe and Vear (1984) with minor modifications (Escande et al. 2002) and immediately covered with paper bags up to 10 days postinoculation (dpi). To check the efficacy of the procedure, a susceptible cultivar was simultaneously inoculated with the tested ILs at all inoculation dates.

Five phenotypic variables were registered: (a) disease incidence (DI), i.e., the number of plants infected over the number of plants inoculated in each row; (b) disease severity (DS), i.e., average proportion of capitulum rotted area of plants inoculated in each row; (c) the area under the disease progress curve for DI (AUDPCI); (d) the area under the disease progress curve for DS (AUDPCS); and (e) incubation period (IP), i.e., average number of days until onset of symptoms in each row. Evaluations were performed at 14, 17, 21, 24, and 28 dpi. Disease assessments were performed by a single person (C. V. Filippi) to avoid interrater error.

DI, DS, AUDPCI, AUDPCS, and IP means per year and inoculation date were calculated and plotted relative to global and annual means, respectively.

Mean, highest, and lowest temperature $\left({ }^{\circ} \mathrm{C}\right)$ and relative humidity (\%) were registered daily by a weather station located $400 \mathrm{~m}$ from the FT. Data can be accessed at http://anterior.inta.gov.ar/balcarce/info/ meteorologia/meteoro2.htm. The mean values of the variables obtained at the different inoculation dates were correlated with temperature and relative humidity using Spearman's rank correlation.

Statistical analysis. Because of the different statistical properties of the phenotypic variables analyzed, appropriate linear or generalized models were chosen accordingly. All statistical analyses were conducted using InfoStat (Di Rienzo et al. 2014). Models are described for each variable below. Random terms are represented using at least one Latin letter.

Disease incidence. This variable measures the proportion of diseased plants relative to the total number of plants exposed. It can be treated as a binomial count, with the following model being considered as suitable:

$$
\log \left(\frac{\pi_{i j k l}}{1-\pi_{i j k l}}\right)=\mu+\lambda_{i}+c_{j}+f_{k j}+\lambda f_{i k j}+b_{l j}
$$

where $\pi_{i j k l}$ represents the probability of a plant becoming infected if it belongs to the inbred line $i$, evaluated in field trial $j$, inoculated at date $k$ in field trial $j$, and located in block $l$ in field trial $j$. The terms $\lambda_{i}$, $c_{j}, f_{k j}, b_{l j}$, and $\lambda f_{i j k}$ refer to the effects of the inbred line $i$, field trial $j$, inoculation date $k$ at field trial $j$, and block $l$ within trial $j$. Finally, $\lambda f_{i j k}$ refers to the interaction between IL and date of inoculation. Common assumptions for random effects apply. 
Disease severity. The DS was visually quantified, by applying a diagrammatic scale developed for SHR by the Plant Pathology group of AES INTA Balcarce (ratio scale 0 to $100 \%$, with $10 \%$ intervals). The generated data were subjected to the square root-arcsine transformation before being fitted to a GLMM, with $Y_{i j k l}$ being the response variable defined as:

$$
Y_{i j k l}=\mu+\lambda_{i}+c_{j}+f_{j k}+\lambda f_{i j k}+b_{l j}+e_{i j k l}
$$

where $Y_{i j k l}$ represents the severity for the inbred line $i$, evaluated in field trial $j$, inoculated at date $k$ in field trial $j$, and located in block $l$ in field trial $j$. All terms have the same meaning as in model (1), except for the additional term $e_{i j k l}$ that represents the classical normal error term.

Area under the disease progress curve. The estimation of the area under the disease progress curve for DI and DS (AUDPCI and AUDPCS) was carried out based on all data collected at 14, 17, 21, 24, and 28 dpi, using the formula described in Shaner and Finney (1977):

$$
\sum_{i=1}^{n}\left(\frac{x_{i+1}+x_{i}}{2}\right)\left(T_{i+1}-T_{i}\right)
$$

where $x_{i}$ is the proportion of diseased plants or proportion of capitulum rotted area at time $T_{i},\left(T_{i+1}-T_{i}\right)$ represents the time (days) between two successive observations, and $n$ is the total number of observations. AUDPCI was estimated using the data from all five FTs, whereas AUDPCS was estimated using data from the first four FTs, because of the lack of DS data for all days post inoculation (i.e., 14, 17, 21, 24 , and $28 \mathrm{dpi}$ ) for the 2013-14 FT. After the normality assumption was verified, the adjusted means for these variables were obtained by fitting to model as described above (2).

Incubation period. Adjusted incubation period means were obtained from a model as described above (2), after the normality assumption was verified.

For all phenotypic variables, means of ILs were subjected to multiple comparison tests using the DGC procedure (Di Rienzo et al. 2002). Finally, the models described above were refitted, considering the IL effect as random for estimating the contribution of the genotype to the phenotypic variance (i.e., broad-sense heritability, $\mathrm{H}^{2}$ ).

Principal component and cluster analyses. Principal component analysis (PCA) was carried out using the standardized adjusted means of all five evaluated phenotypic variables as implemented in InfoStat (Di Rienzo et al. 2014).

Cluster assignment and selection of optimal number of clusters were done using the R package mclust (Fraley and Raftery 2002, 2007). The model was assumed to be a mixture of diagonal, varying volume and equal shape multivariate normal distributions (VEI model). In addition, a dendrogram was obtained from a matrix of $\mathrm{Eu}-$ clidean distances calculated from the standardized phenotypic means using the unweighted pair-group method (UPGMA).

\section{Results}

We conducted a 5-year field experiment to evaluate the resistance of 137 sunflower ILs to $S$. sclerotiorum. The overall mean of inoculated plants per experimental unit was 15 . The number of ILs included in each FT was variable: 69 in $2009-10,69$ in 2010-11, 112 in 2011-12, and 137 in 2012-13 and 2013-14.

Controlled inoculation produced the typical disease symptoms. The lesions increased rapidly during the first weeks of observation to reach a plateau at $28 \mathrm{dpi}$, and therefore we selected this time point as the final stage of the disease.

The FTs showed differences in disease levels, regardless of the phenotypic variable under analysis. The values of DI, DS, and AUDPCI were above the overall mean in 2009-10 and 2012-13 and below it in 2011-12 and 2013-14, with values for 2010-11 showing a different behavior depending on the variable (Fig. 1A to C). AUDPCS showed the same pattern as DS, except for the 2009-10 FT, in which the AUDPCS was below. the overall mean (Fig. 1B and D). In addition, seasons with lower DI, DS, and AUDPCI had longer IP than those with higher values (Fig. 1E). DI and DS progress curves are presented in Supplementary Figs. S1 and S2.
Due to the differences in flowering time among the inbred lines included in this study, each FT involved several inoculation dates, with different sets of lines being inoculated on each date. Figure 2 shows the behavior of the five phenotypic variables for the set of lines inoculated along successive dates relative to the overall mean of the corresponding FT. Although all inoculation dates were suitable to produce disease, a number of them showed large deviations from FT means (Fig. 2). For example, the last five inoculation dates of 2010-11 FT showed DI and AUDPCI levels well below the mean (Fig. 2A and C). Based on these observations, we included inoculation date as a random effect in our statistical models.

Significant negative correlations were found between temperature on the inoculation date (maximum, minimum, and mean) and DI, DS, and AUDPCI, while no correlation was observed with AUDPCS. In contrast, temperature was positively correlated with IP (Spearman's rank correlation test, $P<0.05$ ). No variable was correlated with relative humidity (Supplementary Table S2).

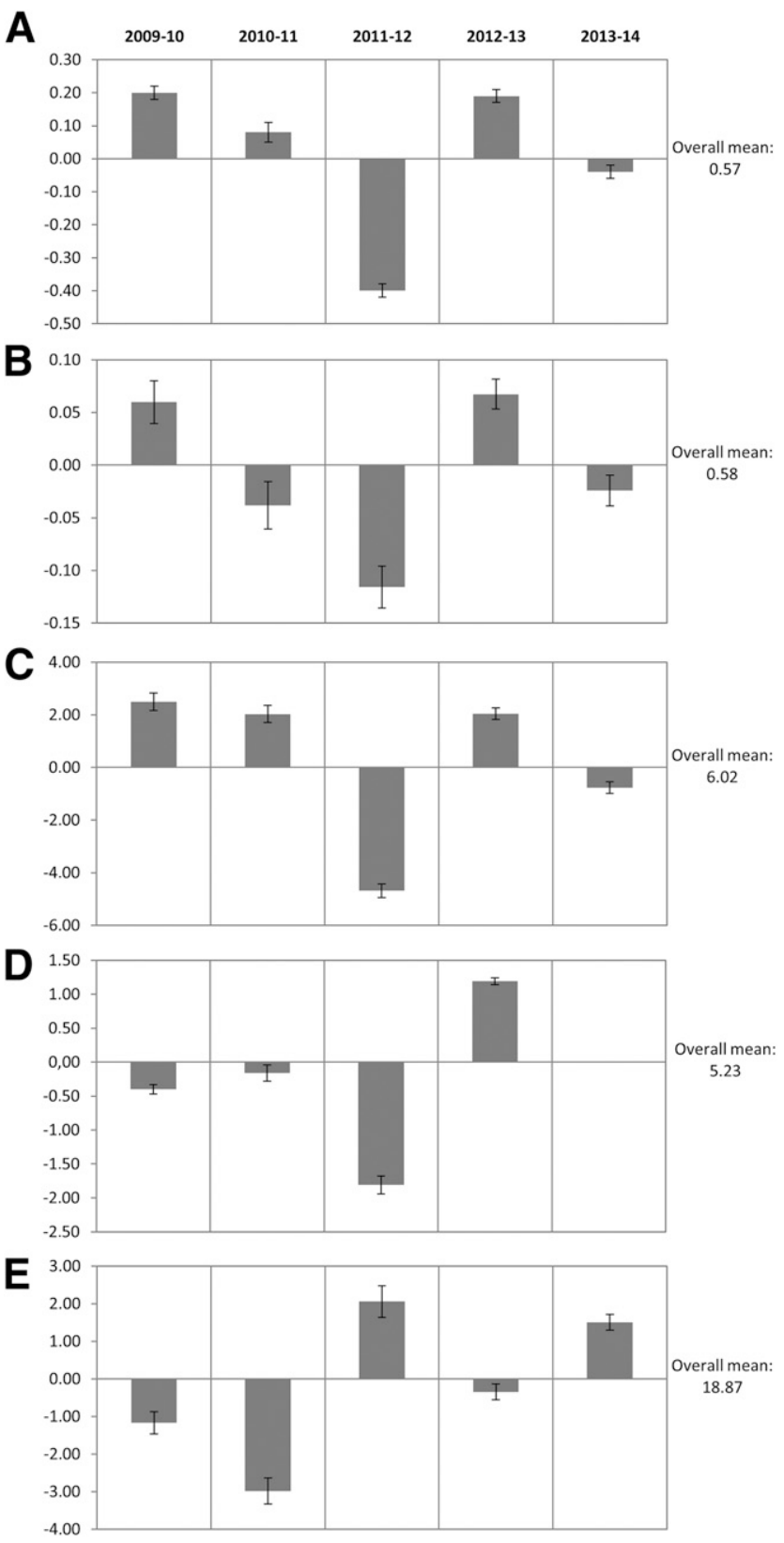

Fig. 1. Annual means of Sclerotinia head rot-related phenotypic variables relative to the overall mean. $\mathbf{A}$, disease incidence; $\mathbf{B}$, disease severity; $\mathbf{C}$, area under the disease progress curve for disease incidence; $\mathbf{D}$, area under the disease progress curve for disease severity; $\mathbf{E}$, incubation period. Horizontal lines represent the overall mean. Vertical brackets represent standard errors. 


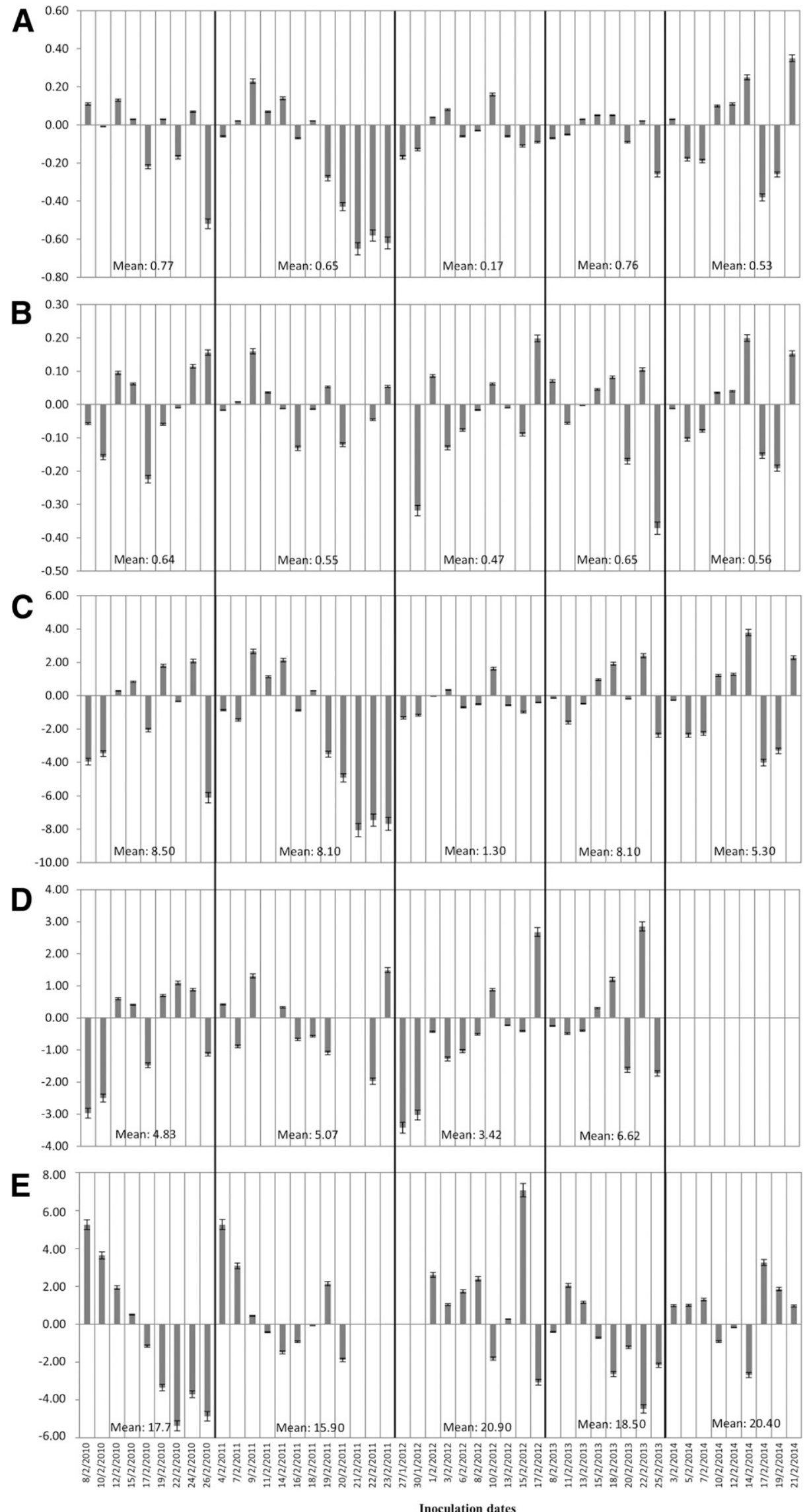

Fig. 2. Means of Sclerotinia head rot-related phenotypic variables per inoculation date relative to their respective annual means. A, disease incidence; B, disease severity; C, area under the disease progress curve for disease incidence; D, area under the disease progress curve for disease severity (data for the 2014-15 field trial is not available); E, incubation period. Horizontal lines represent the annual means. 
Statistical analysis of SHR-related phenotypic variables. The adjusted means for DI, DS, AUDPCI, AUDPCS, and IP were estimated by applying mixed-effect models (1) and (2) (Supplementary Table S3a). The overall adjusted mean (and range) at 28 dpi was 0.55 (0.13 to 1) for DI, $0.58(0.22$ to 0.88$)$ for DS, 5.86 ( 0.92 to 11.21$)$ for AUDPCI, and 4.76 (0.14 to 8.99) for AUDPCS (Fig. 3A to D). The overall mean of IP was 18.92 dpi, ranging from 14.23 to 24.80 (Fig. $3 \mathrm{E})$. Statistically significant differences were observed among ILs for all variables $(P<0.001)$. The DGC test (Di Rienzo et al. 2014) classified the ILs into two different groups according to DI, DS, AUDPCI, AUDPCS, and IP, respectively (Supplementary Table S4).

Taken together, the results of the five variables suggest that the ILs ALB2/5261, 5383, 51084/5429, and 7-1-1 (from the sunflower breeding program of INTA) and the public IL RK416 are moderately resistant to SHR. On the other hand, the ILs R459-4, B485-5, R4633, R467-3, 5289, 5431, and B481-3 (from the sunflower breeding

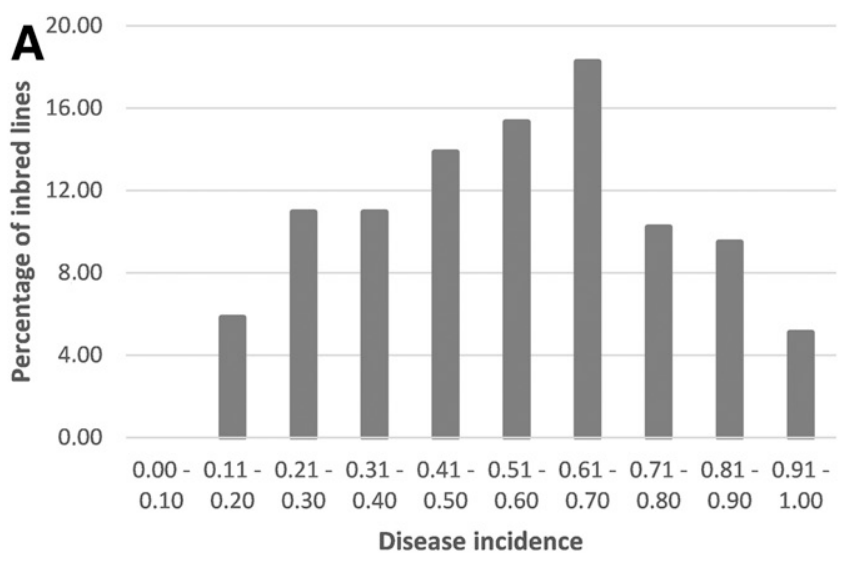

C

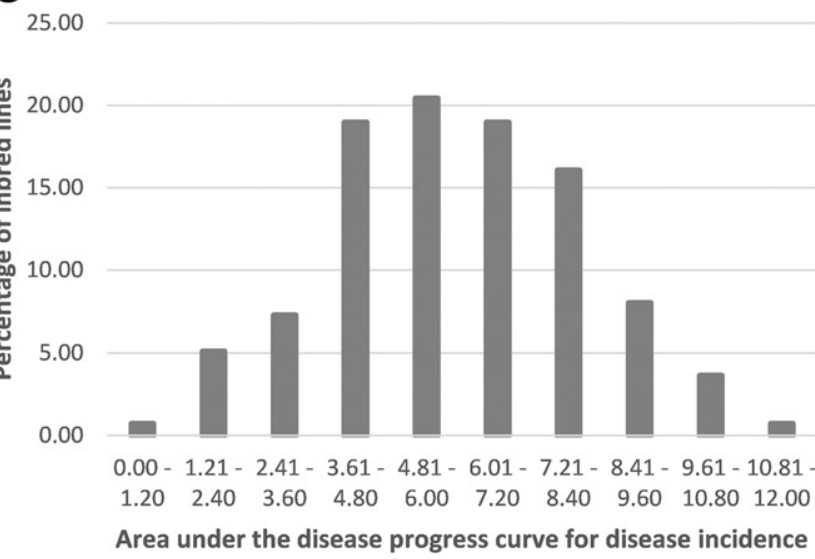

E 30.00

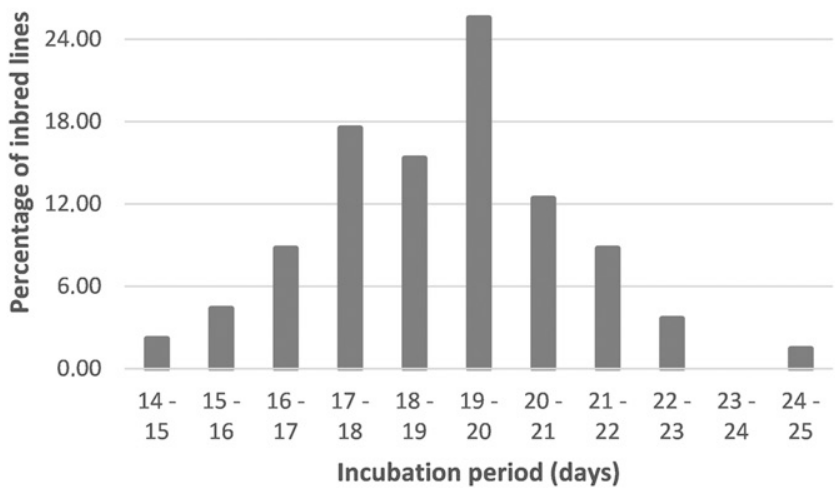

program of INTA) are highly susceptible to SHR. The phenotypic response of these ILs was consistent across FTs, with the exception of ILs 5383 and 51084/5429 on the 2009-10 FT, which showed DI values above the FT mean ( 0.875 and 0.78 versus 0.77 ), and RK416 on the 2012-13 FT, which showed DI and AUCPCI values slightly above the FT mean ( 0.825 versus 0.76 and 8.24 versus 8.1 , respectively).

Significant positive correlations were found between the adjusted means of DI, DS, AUDPCI, and AUDPCS, whereas IP was significantly negatively correlated with the other four variables (DI, DS, AUDPCI, and AUDPCS; Table 1).

The heritabilities of DI, DS, AUDPCI, AUDPCS, and IP for each FT and across the FTs were estimated by applying random effect models (Supplementary Table S3b). A broad distribution of $\mathrm{H}^{2}$ values was obtained across FTs, from high heritability estimates (e.g.: DI 2009-10 FT, $\mathrm{H}^{2}=96.58 \%$ ) to values near 0 (e.g.: DS 2011-12 FT, $\mathrm{H}^{2}=0.45 \%$ ). When all the FTs were considered together, the five phenotypic

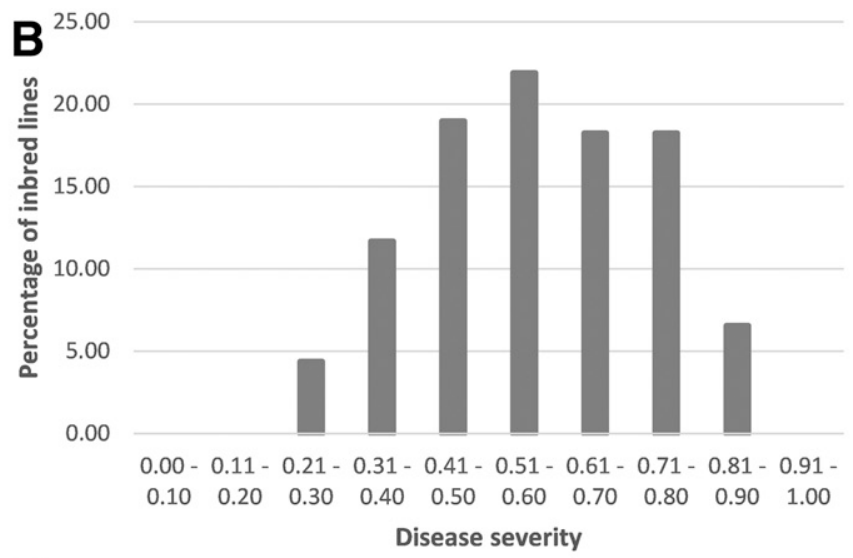

D

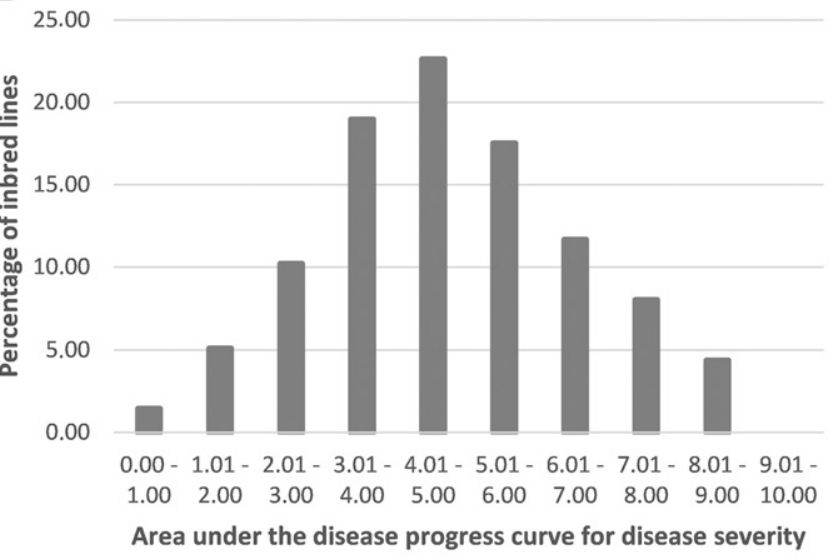

Fig. 3. Frequency histograms of Sclerotinia head rot-related phenotypic variables $(n=137)$. A, Disease incidence; $\mathbf{B}$, disease severity; $\mathbf{C}$, area under the disease progress curve for disease incidence; D, area under the disease progress curve for disease severity (data for the 2014-15 field trial is not available); E, incubation period. 
variables under study showed moderate heritability, with DS having the highest $\left(\mathrm{H}^{2}=20.64 \%\right)$ and IP the lowest values $\left(\mathrm{H}^{2}=10.58 \%\right)$ (Supplementary Table S5).

Principal component and cluster analyses. A PCA was carried out using the model-based adjusted means of the phenotypic variables (Fig. 4). The first two axes explained 74.8 and $14.0 \%$ of the total variation, respectively. All variables were positively correlated with PC 1, except for IP. AUDPCI showed the highest correlation with this axis (0.94). All variables were positively correlated with PC 2, except for DS. DI showed the highest correlation with this axis (0.59). The ILs are widely scattered throughout the PCA graph. The most resistant ILs appear on the left of the upper-left quadrant of the PCA biplot, indicating lower DI, DS, AUDPCI, and AUDPCS and higher IP. Again, ALB2/5261 and 5383 responded better against SHR, followed by $51084 / 5429$. The VEI model in the mclust package split the 137 ILs into six groups. Means and standard deviations of all variables for each group are provided in Supplementary Table S6.

The Euclidean distances derived from model-based standardized means varied from 0.19 to 9.35 , with an average of 2.78. A dendrogram depicting the relationships among ILs is provided in Supplementary Fig. S3.

\section{Discussion}

Sunflower has extensive phenotypic and molecular diversity that can be exploited in breeding for SHR resistance. However, evaluation of SHR is a challenging task that involves the selection of the inoculation method, disease descriptors, and realistic statistical approaches for modeling the data. Moreover, knowledge of the heritability of the trait is needed to aid in the decision-making process for SHR resistance breeding.

Table 1. Spearman's correlation analysis of Sclerotinia head rot-related variables. Coefficients (below diagonal) and $P$-values (above diagonal). ${ }^{a}$

\begin{tabular}{llcccc}
\hline & DI & DS & AUDPCI & AUDPCS & IP \\
\hline DI & 1 & $<0.0001$ & $<0.0001$ & $<0.0001$ & $<0.0001$ \\
DS & 0.51 & 1 & $<0.0001$ & $<0.0001$ & $<0.0001$ \\
AUDPCI & 0.9 & 0.72 & 1 & $<0.0001$ & $<0.0001$ \\
AUDPCS & 0.47 & 0.85 & 0.67 & 1 & $<0.0001$ \\
IP & -0.51 & -0.77 & -0.73 & -0.71 & 1 \\
\hline
\end{tabular}

${ }^{a}$ DI: disease incidence; DS: disease severity; AUDPCI: area under the disease progress curve for DI; AUDPCS: area under the disease progress curve for DS; IP: incubation period.
Controlled inoculation is now recognized as the method of choice for disease assessment since the degree of SHR infection is affected by weather conditions and the presence of sclerotia in the soil, two factors prone to regional and temporal variation (Vear and Tourvieille de Labrouhe 1984). The ascospore method, developed by Vear and Tourvieille de Labrouhe (1984), is one of the most employed testing procedures for screening sunflower lines and hybrids, as it allows measurement of host resistance from the beginning of the infection process. This method requires a careful determination of the optimal inoculum concentration since a high inoculum pressure must be applied to ensure a sufficient number of infected plants. In the present study, SHR phenotypic variables showed a wide range of variation indicating that the used inoculum concentration was adequate to accurately measure disease.

Previous studies investigating the influence of sunflower genotypes and S. sclerotiorum isolates on SHR response reported that both variables exerted highly significant effects, while they did not interact with each other (Thuault and Tourvieille de Labrouhe 1988; Vear 2004). No changes in the resistance ranking of sunflower genotypes were observed when testing $S$. sclerotiorum isolates with different levels of aggressiveness. Thus, the authors concluded that breeding and disease resistance tests with any isolate or population of $S$. sclerotiorum should be valid for all areas and many years (Vear 2004). These observations suggest that sunflower has "horizontal" and "race non-specific" resistance to S. sclerotiorum and that the use of a mixture of spores of different origins and natural populations is probably the safest way to ensure the long-term stability of the results (Vear 2004). In the present work, the inoculation of capitula using a mixture of ascospores derived from sclerotia found in naturally infected plants allowed a clear discrimination of responses for the five phenotypic variables, i.e., disease incidence (DI), disease severity (DS), AUDPC incidence (AUDPCI), AUDPC severity (AUDPCS), and incubation period (IP).

In our experiments, year-to-year differences in temperature on inoculation dates seem to have been associated with disease occurrence. Indeed, temperature on inoculation date was significantly correlated with four of the phenotypic variables evaluated (i.e., DI, DS, AUDPCI, and IP), while the inoculation date effect explained a considerable proportion of random variation in our GLMMs. The lowest infection levels occurred in 2011-12, which showed the highest temperatures (mean maximum and minimum temperatures of 35.6 and $23.3^{\circ} \mathrm{C}$, respectively). In contrast, the highest disease levels occurred during the 2009-10 FT, when temperatures where the

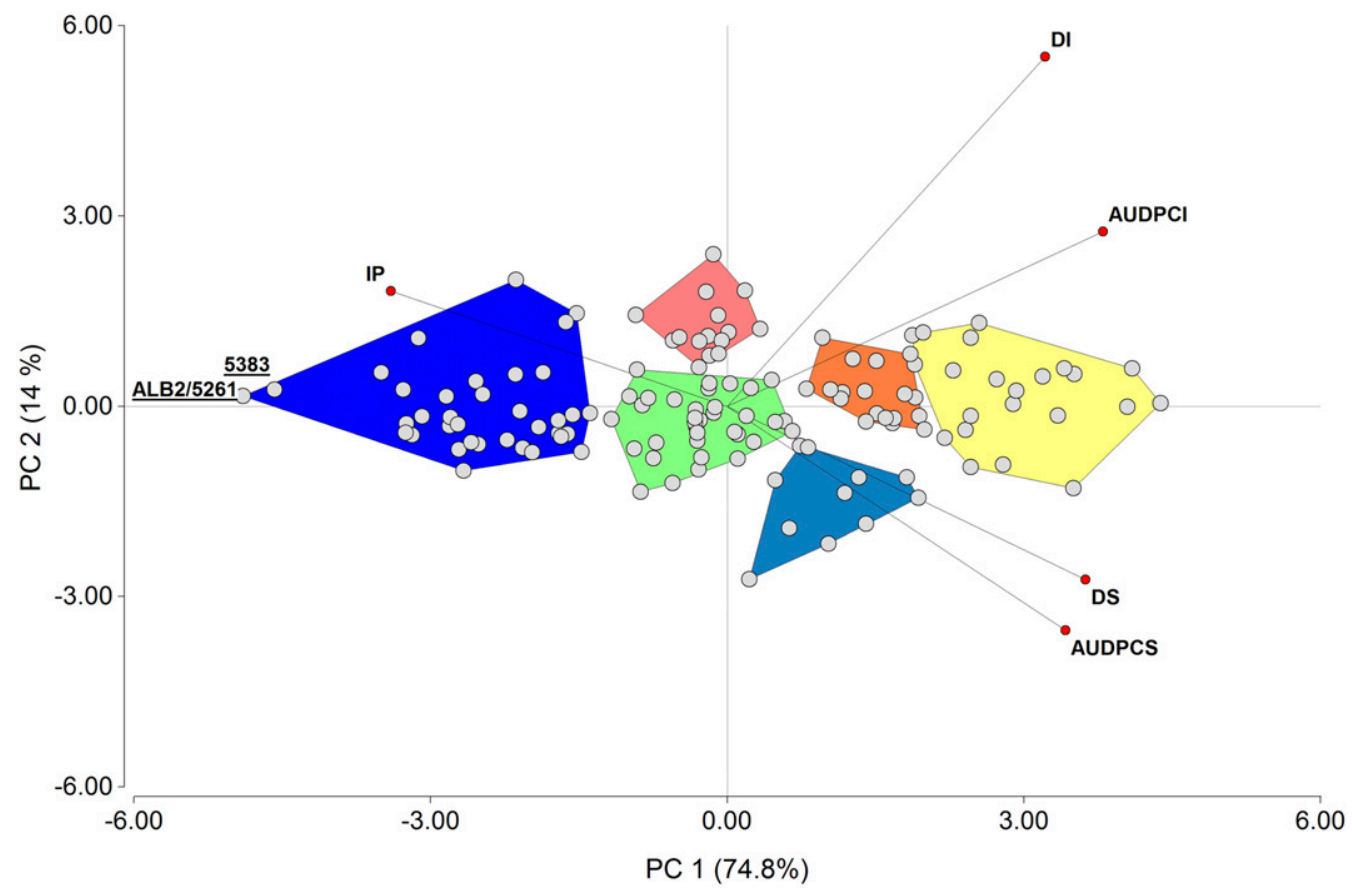

Fig. 4. PCA biplot based on the matrix of the adjusted means of the Sclerotinia head rot-related phenotypic variables. Points represent inbred lines (ILs). Variables are indicated by lines extending from the center of the graph. The ILs were colored based on their mclust VEI group assignment. The most resistant ILs are underlined. 
lowest (mean maximum and minimum temperatures of 32 and $21.8^{\circ} \mathrm{C}$, respectively). Taking into account the influence of temperature on the disease development, Tourvieille de Labrouhe and Vear (1984) recommended that only materials inoculated on the same day should be used for comparisons between different sunflower accessions. The protocol of ascospore inoculation requires capitula to be inoculated at the R5.2 stage (Schneiter and Miller 1981), but the high variability in flowering time of sunflower precludes the simultaneous treatment of a large number of ILs. In the past, this problem was partially solved by expressing phenotypic measures relative to a susceptible control inoculated on the same day (Bert et al. 2002, 2004; Castaño et al. 1993; Vear 2004). Under this approach, control individuals are planted on staggered dates so that flowering controls are available throughout the flowering period. In practice, however, this method is time and resource consuming. In this work, different GLMM models were tested and applied to estimate the adjusted means of DI, DS, AUDPCI, AUDPCS, and IP. Using the mixed model approach, we were able to not only deal with complex data, but also to solve the problem of different inoculation dates by including the random effect of the inoculation date in the statistical models. This precluded the necessity of including controls to adjust the phenotypic measures and allowed us to test genotype-by-environment interactions (GE, i.e., the differential genotypic response to different environments).

The DI is regarded as suitable for estimating resistance to fungal penetration, while the IP and the DS are considered to be a measure of resistance to the spread of the fungus within the host tissues (Gentzbittel et al. 1998). DI is relatively objective and easy to obtain, making it suitable for scoring a large number of data, particularly when these are collected by non-experts (Madden and Hughes 1995). In turn, DS is used to characterize fungal diseases affecting specific plant tissues (Kranz 1988), as it most adequately describes the spatio-temporal dynamics in terms of disease increase and spread (Madden and Campbell 1990). Besides the final symptoms, the actual disease progression over time, as measured by AUDPCI and AUDPCS, is also necessary for a more in-depth analysis of the disease. AUDPC has gained increasing importance for the measurement of quantitative disease resistance in most foliar pathosystems (Jeger and Viljanen-Rollinson 2001) and it is currently applied to soil-borne diseases (Pouralibaba et al. 2015). Indeed, the analysis of AUDPCI and AUDPCS, in combination with maximum DI and DS, enabled a more robust characterization of the resistance response to rice blast (Long et al. 2000), tomato bacterial wilt (Rivard et al. 2012), apple brown rot (Holb et al. 2012), and Phytophthora crown and root rot (Foster and Hausbeck 2010).

In this study, individual and combined analyses of the five disease variables revealed that the ILs examined here show a wide range of responses to SHR, with ALB2/5261 and 5383 appearing as the most resistant. Moreover, we found significant positive correlations among DI, DS, AUDPCI, and AUDPCs and negative correlations between IP and the other four variables (DI, DS, AUDPCI, and AUDPCS). The correlation coefficients ranged from moderate to high but did not approach unity, indicating that the different variables should still be considered as separate dimensions of the expression space of the disease. The correlation value obtained between DI and DS $(r=0.55)$ was similar to that reported for sunflower by Yue et al. (2008) ( $\mathrm{r}$ between 0.57 and 0.64). The negative correlation between IP and DI, DS, AUDPCI, and AUDPCS mentioned above suggests that incubation time is shortened in years favorable for disease development, and vice versa. In sunflower, similar correlation values were reported by Bert et al. (2002) ( $r$ between -0.293 and -0.477 ) and Bert et al. (2004) $(\mathrm{r}=0.55)$.

In addition to measuring correlations among disease variables, some authors have investigated the relationship between SHR and morphological or field characters, as a proxy for disease resistance (Castaño et al. 1993; Hahn 2002). Hahn (2002) found that the physiological stage does not influence the results of head rot tests. Although inbred lines showed significant differences for days of flowering, no correlations were observed between this variable and resistance measures. These results support the proposals of Castaño et al. (1993) that taking into account morphological and field characters will not improve the efficiency of breeding programs for sunflower SHR.

Another key aspect in plant breeding is the determination of the trait's heritability. Broad-sense heritability $\left(\mathrm{H}^{2}\right)$ expresses the extent to which individuals' phenotypes are determined by the genotypes (Falconer et al. 1996). In the breeding context, phenotypic selection would be efficient for high heritability traits, while marker-assisted selection, via the biparental or the AM approach, appears as the best option for low heritability traits. In this work, heritability was estimated by pooling data not only from the two replicates of the five FTs, but also from the two replicates of each FT to obtain a single mean for each variable. When the results of all FTs were taken together, the five phenotypic variables showed moderate heritability, with $\mathrm{H}^{2}$ ranging between 20 and $10.58 \%$ (DS and IP, respectively). In turn, when each FT was analyzed separately, $\mathrm{H}^{2}$ showed a broader distribution, varying from high to very low heritability values. The broad distribution of $\mathrm{H}^{2}$ values resulting from the separate analysis of each FT is similar to the estimates obtained by previous authors for DI, DS, IP, and other SHR-related phenotypic variables (Bert et al. 2002, 2004; Mestries et al. 1998; Zubrzycki 2014).

Given that $S$. sclerotiorum is present in almost all sunflower growing regions of the world, one of the main goals of SHR breeding is to ensure the long-term stability and broad usefulness of the desired characteristics. In the present study, the phenotypic response of the moderately resistant ILs was consistent across FTs. Moreover, sunflower public lines described as resistant by American and French research teams (e.g., Mestries et al. 1998; Yue et al. 2008) behaved as resistant in our geographic area and with a local inoculum challenge. In this context, it is expected that SHR results from evaluations performed at AES INTA Balcarce will hold in different environments.

In comparison with previous screenings for SHR resistance in sunflower (e.g., Castaño et al. 1993; Hahn 2002; Vear and Tourvieille de Labrouhe 1988), our phenotypic characterization relies on a larger set of ILs, new disease variables, and more appropriate and realistic statistical approaches for modeling the data. Despite the fact that no complete resistance was detected, our results reinforce the notion that different phenotypic variables are required to fully capture disease response. In this context, the ILs ALB2/5261 and 5383 emerge as the best candidates for breeding based on their lower DI, DS, AUDPCI, and AUDPCS, and their higher IP values. The ample phenotypic variability of our collection, along with the moderate heritability estimates, highlight the importance of molecular breeding approaches to gain new insights into the genetic basis of sunflower resistance to SHR.

\section{Acknowledgments}

This research was supported by Instituto Nacional de Tecnología Agropecuaria (PNBIO-1131042, 1131043) and Agencia Nacional de Promoción Científica y Técnica (PICT2011 1365). We thank Silvio Giuliano and Carlos Antonelli for their field assistance at INTA Balcarce. We are also grateful to Dr. Silvia Pietrokovsky, who kindly revised the English of the manuscript. Finally, we wish to thank the editor and reviewers for the valuable comments and suggestions that have greatly improved this manuscript.

\section{Literature Cited}

Baldini, M., Turi, M., Vischi, M., Vannozzi, G. P., and Olivieri, A. M. 2002. Evaluation of genetic variability for Sclerotinia sclerotiorum Lib. de Bary resistance in sunflower and utilization of associated molecular markers. Helia 25:177-189.

Bert, P.-F., Dechamp-Guillaume, G., Serre, F., Jouan, I., De Labrouhe, D. T., Nicolas, P. and Vear, F. 2004. Comparative genetic analysis of quantitative traits in sunflower (Helianthus annuus L.) 3. Characterization of QTL involved in resistance to Sclerotinia sclerotiorum and Phoma macdonaldi. Theor. Appl. Genet. 109:865-874.

Bert, P.-F., Jouan, I., De Labrouhe, D. T., Serre, F., Nicolas, P., and Vear, F. 2002. Comparative genetic analysis of quantitative traits in sunflower (Helianthus annuus L.) 1. QTL involved in resistance to Sclerotinia sclerotiorum and Diaporthe helianthi. Theor. Appl. Genet. 105:985-993.

Boland, G. J., and Hall, R. 1994. Index of plant hosts of Sclerotinia sclerotiorum. Can. J. Plant Pathol. 16:93-108.

Castaño, F., and Giussani, M. 2009. Effectiveness of components of partial resistance in assessing white rot of sunflower head. Helia 32:59-68.

Castaño, F., Vear, F., and Tourvieille de Labrouhe, D. 1993. Resistance of sunflower inbred lines to various forms of attack by Sclerotinia sclerotiorum and relations with some morphological characters. Euphytica 68:85-98.

Clarkson, J. P., Coventry, E., Kitchen, J., Carter, H. E., and Whipps, J. M. 2013. Population structure of Sclerotinia sclerotiorum in crop and wild hosts in the UK. Plant Pathol. 62:309-324.

Di Rienzo, J. A., Casanoves, F., Balzarini, M. G., Gonzalez, L., Tablada, M., and Robledo, C. 2014. Infostat versión 2014. http://www.infostat.com.ar.

Di Rienzo, J. A., Guzmán, A. W., and Casanoves, F. 2002. A multiple comparison method based on the distribution of the root node distance of a binary tree 
obtained by average linkage of the matrix of euclidean distances between treatment means. J. Agric. Biol. Environ. Stat. 7:129-142.

Escande, A. R., Laich, F. S., and Pedraza, M. V. 2002. Field testing of honeybeedispersed Trichoderma spp. to manage sunflower head rot (Sclerotinia sclerotiorum). Plant Pathol. 51:346-351.

Falconer, D. S., Mackay, T. F., and Frankham, R. 1996. Introduction to quantitative genetics. Pearson Education Limited, Essex, England.

Filippi, C. V., Aguirre, N., Rivas, J. G., Zubrzycki, J., Puebla, A., Cordes, D., Moreno, M. V., Fusari, C. M., Alvarez, D., Heinz, R. A., Hopp, H. E., Paniego, N. B., and Lia, V. V. 2015. Population structure and genetic diversity characterization of a sunflower association mapping population using SSR and SNP markers. BMC Plant Biol. 15:52.

Foster, J. M., and Hausbeck, M. K. 2010. Resistance of pepper to Phytophthora crown, root, and fruit rot is affected by isolate virulence. Plant Dis. 94:24-30.

Fraley, C., and Raftery, A. E. 2002. Model-based clustering, discriminant analysis, and density estimation. J. Am. Stat. Assoc. 97:611-631.

Fraley, C., and Raftery, A. E. 2007. Bayesian regularization for normal mixture estimation and model-based clustering. J. Classif. 24:155-181.

Fusari, C. M., Di Rienzo, J. A., Troglia, C., Nishinakamasu, V., Moreno, M. V., Maringolo, C., Quiroz, F., Alvarez, D., Escande, A., Hopp, E., Heinz, R., Lia, V. V., and Paniego, N. B. 2012. Association mapping in sunflower for Sclerotinia head rot resistance. BMC Plant Biol. 12:93.

Gentzbittel, L., Mouzeyar, S., Badaoui, S., Mestries, E., Vear, F., Tourvieille de Labrouhe, D., and Nicolas, P. 1998. Cloning of molecular markers for disease resistance in sunflower, Helianthus annuus L. Theor. Appl. Genet. 96:519-525.

Hahn, V. 2002. Genetic variation for resistance to Sclerotinia head rot in sunflower inbred lines. F. Crop. Res. 77:153-159.

Holb, I. J., Balla, B., Vámos, A., and Gáll, J. M. 2012. Influence of preharvest calcium applications, fruit injury, and storage atmospheres on postharvest brown rot of apple. Postharvest Biol. Technol. 67:29-36.

Jeger, M. J., and Viljanen-Rollinson, S. L. H. 2001. The use of the area under the disease-progress curve (AUDPC) to assess quantitative disease resistance in crop cultivars. Theor. Appl. Genet. 102:32-40.

Kranz, J. 1988. Measuring plant disease. Pages 35-50 in: Experimental Techniques in Plant Disease Epidemiology. Springer, Berlin/Heidelberg, Germany.

Long, D. H., Lee, F. N., and TeBeest, D. O. 2000. Effect of nitrogen fertilization on disease progress of rice blast on susceptible and resistant cultivars. Plant Dis. 84:403-409.

Madden, L. V., and Campbell, C. L. 1990. Nonlinear disease progress curves. Epidemics Plant Dis. 13:181-229.

Madden, L. V., and Hughes, G. 1995. Plant disease incidence: distributions, heterogeneity, and temporal analysis. Annu. Rev. Phytopathol. 33:529-564.

Mestries, E., Gentzbittel, L., Tourvieille de Labrouhe, D., Nicolas, P., Vear, F., and Am, S. 1998. Analyses of quantitative trait loci associated with resistance to Sclerotinia sclerotiorum in sunflowers (Helianthus annuus L.) using molecular markers. Mol. Breed. 4:215-226.
Moreno, M. V., Nishinakamasu, V., Loray, M., Alvarez, D., Gieco, J., Vicario, A. Hopp, H. E., Heinz, R. A., Paniego, N., and Lia, V. V. 2013. Genetic characterization of sunflower breeding resources from Argentina: assessing diversity in key open-pollinated and composite populations. Plant Genet. Resour. 11:238-249.

Pouralibaba, H. R., Rubiales, D., and Fondevilla, S. 2015. Identification of resistance to Fusarium oxysporum f.sp. lentis in Spanish lentil germplasm. Eur. J. Plant Pathol. 143:399-405.

Rivard, C. L., O'Connell, S., Peet, M. M., Welker, R. M., and Louws, F. J. 2012 Grafting tomato to manage bacterial wilt caused by Ralstonia solanacearum in the southeastern United States. Plant Dis. 96:973-978.

Rönicke, S., Hahn, V., Vogler, A., and Friedt, W. 2005. Quantitative trait loci analysis of resistance to Sclerotinia sclerotiorum in sunflower. Phytopathology 95 834-839.

Schneiter, A. A., and Miller, J. F. 1981. Description of sunflower growth stages. Crop Sci. 21:901-903.

Shaner, G., and Finney, R. E. 1977. The effect of nitrogen fertilization on the expression of slow-mildewing resistance in knox wheat. Phytopathology 67: 1051-1056.

Thuault, M. C., and Tourvieille de Labrouhe, D. 1988. Etudes du pouvoir pathogene de huit isolats de Sclerotinia appartenant aux especes Sclerotinia sclerotiorum, Sclerotinia minor et Sclerotinia trifoliorum sur le tournesol. Int. Tech. CETIOM. 103:21-27.

Tourvieille de Labrouhe, D., and Vear, F. 1984. Comparaison de méthodes d'estimation de la résistance du tournesol à Sclerotinia sclerotiorum (Lib.) de Bary. Agronomie 4:517-525.

Van, B., and Miller, J. F. 2004. Methods of inoculation of sunflower heads with Sclerotinia sclerotiorum. Helia 27:137-142.

Vear, F. 2004. Reaction of sunflower lines to a series of Sclerotinia sclerotiorum isolates. Page 15 in: 16th International Sunflower Conference, Fargo, ND, U.S.A.

Vear, F., and Tourvieille de Labrouhe, D. 1984. Recurrent selection for resistance to Sclerotinia sclerotiorum in sunflowers using artificial infections. Agronomie 4:789-794.

Vear, F., and Tourvieille de Labrouhe, D. 1988. Heredity of resistance to Sclerotinia sclerotiorum in sunflowers. II. - Study of capitulum resistance to natural and artificial ascospore infections. Agronomie 8:503-508.

Yue, B., Radi, S. A., Vick, B. A., Cai, X., Tang, S., Knapp, S. J., Gulya, T. J. Miller, J. F., and Hu, J. 2008. Identifying quantitative trait loci for resistance to Sclerotinia head rot in two USDA sunflower germplasms. Phytopathology 98:926-931.

Zhu, T., and Salmeron, J. 2007. High-definition genome profiling for genetic marker discovery. Trends Plant Sci. 12:196-202.

Zubrzycki, J. 2014. Estudio de la resistencia a Sclerotinia sclerotiorum en líneas endocriadas de girasol. Ph.D. Thesis, Universidad de Buenos Aires, Ciudad Autónoma de Buenos Aires, Argentina. 